\title{
Primary pericardial mesothelioma presenting as tuberculous pericarditis
}

\author{
D.S.C. Rose', W.T. Vigneswaran ${ }^{2}$, B.A. Bovill ${ }^{3}$, J.F. Riordan ${ }^{4}$, R.N. Sapsford ${ }^{2}$ \\ and R.DeL. Stanbridge ${ }^{2}$ \\ ${ }^{1}$ Department of Histopathology, ${ }^{2}$ Department of Cardiothoracic Surgery, St Mary's Hospital, \\ Praed Street, London W2 INY, ${ }^{3}$ Whipps Cross Hospital, London and ${ }^{4}$ Department of Chest Medicine, \\ Central Middlesex Hospital, London, UK.
}

\begin{abstract}
Summary: Ante-mortem diagnosis of primary pericardial mesothelioma is very rare. We report a case which presented clinically as tuberculous constrictive pericarditis. The patient underwent pericardial resection with an immediate haemodynamic benefit, although the malignant process progressed, and he died 14 weeks later.
\end{abstract}

\section{Introduction}

Malignant mesotheliomas may arise in the pleura $(70-75 \%)$, the peritoneum $(20-25 \%)$, the pericardium $(4 \%)$, or, very rarely, the tunica vaginalis. ${ }^{1}$ In all sites, the symptoms and signs are related to infiltration of adjacent structures, malignant effusion, and ultimately obliteration of the serous cavity. Metastatic spread is usually a clinically minor problem. We present the case of a patient who reported a previous diagnosis of pericardial tuberculosis, and presented with signs of constrictive pericarditis, but who was found to have a primary pericardial mesothelioma.

\section{Case report}

A 29 year old Pakistani clothing merchant presented with a short history of increasing breathlessness. He said that 4 months previously he had been diagnosed as having pericardial tuberculosis in Pakistan and had been on anti-tuberculous therapy since. He gave no history of exposure to asbestos. He was dyspnoeic with an elevated jugular venous pressure, crepitations in the bases of both lungs, and peripheral oedema. There was left cervical lymphadenopathy. He was admitted and the antituberculous therapy continued with the addition of steroids. Baseline biochemical and haematological

Correspondence: D.S.C. Rose, M.B., B.S., Department of Histopathology, Bland Sutton Institute, The Middlesex Hospital Medical School, Mortimer Street, London W1P 7PN, UK.

Accepted: 11 July 1991 indices showed an albumin of $29 \mathrm{~g} / 1$, total protein $81 \mathrm{~g} / \mathrm{l}$, bilirubin $21 \mu \mathrm{mol} / \mathrm{l}$, and an alkaline phosphatase of $336 \mu \mathrm{mol} / 1$. He had an erythrocyte sedimentation rate of $25 \mathrm{~mm} / \mathrm{h}$. The chest X-ray showed bilateral pleural effusions and the electrocardiogram a low voltage pattern. An echocardiogram showed a grossly thickened pericardium with a small posterior pericardial effusion (Figure 1). A pericardiocentesis was performed under echocardiographic control and $150 \mathrm{ml}$ of blood-stained fluid was removed. However, following the procedure he developed increasing dyspnoea, cyanosis, further elevation of the jugular venous pressure, a tachycardia of $120 / \mathrm{min}$, and a falling systolic pressure to $90 \mathrm{mmHg}$ with a pulsus paradoxus of $20 \mathrm{mmHg}$. At emergency pericardectomy, through a bilateral sub-mammary incision with cardiopulmonary bypass standby, the mobility of the heart was noted to be markedly restricted and the parietal and visceral pericardium irregularly thickened to about $2 \mathrm{~cm}$. The lungs and pleura were normal and bilateral serous effusions were drained. The pericardium was resected, apart from two strips containing the phrenic nerves, and the central venous pressure fell from 18 to $8 \mathrm{mmHg}$.

Histological examination of the resected pericardium showed a neoplasm (Figure 2) composed of pleomorphic spindle and cuboidal cells with abundant cytoplasm, arranged in fasicles, solid nodules with cleft formation, and a few areas with a papillary pattern. The mitosis count was 30 per 10 high power fields (magnification $\times 400$ ) with occasional abnormal mitoses. The tumour cells showed positive staining with CAM5.3, a marker for cytokeratin, and were negative for carcinoembryonic antigen (CEA). Stains for acid mucopolysac- 


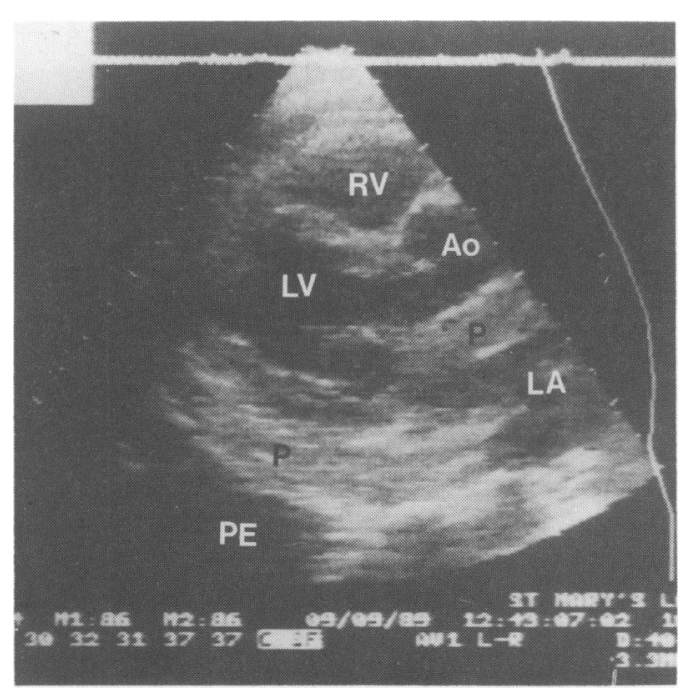

Figure 1 Echocardiogram showing markedly thickened pericardium and pleural effusion. $\mathbf{P}=$ pericardium; $\mathrm{PE}=$ pleural effusion; $\mathrm{RV}=$ right ventricle; $\mathrm{LV}=$ left ventricle; $\mathrm{LA}=$ left atrium; Ao $=$ Aorta.

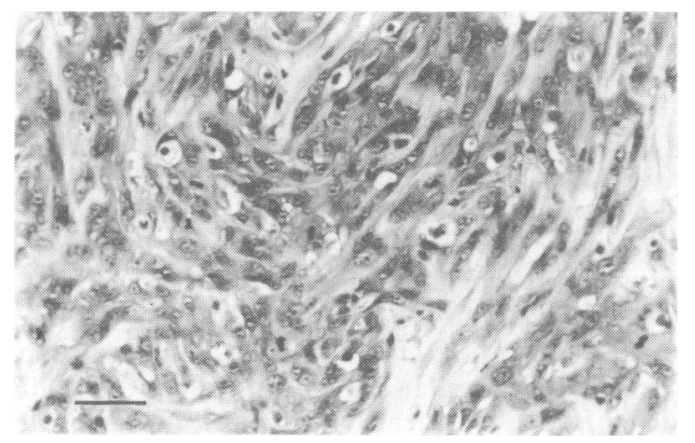

Figure 2 Photomicrograph showing predominal spindle cell area of pericardial malignant mesothelioma. Cellular pleomorphism and several mitotic figures are evident. (Haematoxylin and Eosin) (Bar $=30 \mu \mathrm{m})$.

charides were negative. The appearances were diagnostic of a mesothelioma. Reactive mesothelium, fibrous tissue and normal thymic tissue were also present in the specimens. Ferruginous bodies were not seen in the resected tissue. No features of tuberculosis were found.

At follow-up 6 weeks later his exercise tolerance had improved markedly but subsequently there were signs of further restriction of his cardiac activity. He died 14 weeks after operation. No post-mortem was possible.

\section{Discussion}

Primary pericardial mesotheliomas are rare, with only one diagnosed in 496,795 consecutive hospital admissions, and one in 18,328 autopsies at the Henry Ford Hospital, USA, ${ }^{2}$ and a calculated annual incidence of one in 41 million in Canada. ${ }^{3}$ Pericardial mesothelioma may occur at any age. There are too few published cases for an assessment of aetiological factors. The wide age range and lack of a consistent occupational history preclude a widespread association with asbestos, although there is one report of intrathoracic mesothelioma in a patient who had pericardectomy and application of asbestos for angina pectoris, ${ }^{4}$ and one report of pericardial mesothelioma in a patient with pleural plaques and ferruginous bodies in the lungs. ${ }^{5}$

The histological features of primary mesothelioma of the pericardium do not differ from those of mesothelioma arising in the pleura or elsewhere. Most cases are of a mixed type with both spindle cell and glandular elements. The major differential diagnoses are from a benign reactive proliferation of the mesothelium, in which necrosis, infiltration and cytological atypia are not prominent, and from secondary malignancies, especially adenocarcinoma. Hyaluronidase-labile mucin production is variable, and the most helpful, commonly available immunohistochemical stains are CAM5.2 and CEA. If the latter is negative, a diagnosis of흐 adenocarcinoma is unlikely. ${ }^{6}$

Pericardial mesothelioma can present in such a way that mistaken diagnoses of mitral stenosis in rheumatic fever ${ }^{7}$ and atrial myxoma ${ }^{8}$ have been made, the true nature of the lesion becoming apparent only at operation or post-mortem. The most common presentation is with compromised cardiac function due to pericardial effusion, or due to constriction by an infiltrating mass of tumour. ${ }^{9}$

The differential diagnosis of subacute constrictive pericarditis in a patient from the Indian sub-continent is wide and a common cause is tuberculosis. ${ }^{10}$ Tubercle bacilli are demonstrated in less than half of these cases. One case of pericardial mesothelioma with coexistent pericardial tuberculosis has been reported from India. ${ }^{11}$ We have no independent evidence of tuberculosis in our case. Cultures of pleural an: $\mathbb{1}$ pericardial fluid were negative. Although the history, signs, and echocardiographic findings were consistent with tuberculous pericarditis, persistence or worsening of symptoms and effusion in a patient on effective anti-tuberculous therapy, with steroids, should perhaps alert the clinician to the possibility of another aetiology. As in pleural mesothelioma, primary pericardial mesothelioma is an aggressive disease and in our patient this was responsible for his death within 4 months of diagnosis. 


\section{Acknowledgements}

We should like to thank Dr M. Williams for clinical assistance, S. Lavda and K. Hennessey for the histo-

\section{References}

1. McDonald, J.C. \& McDonald, A.D. Epidemiology of mesothelioma from estimated incidence. Prev Med 1977, 6: 426-446.

2. Fine, G. Primary tumors of the pericardium and heart. Cardiovasc Clin 1973, 5: 208-238.

3. McDonald, A.D., Harper, A., El Attar, G.A. \& McDonald, J.C. Epidemiology of primary malignant mesothelial tumors in Canada. Cancer 1970, 26: 914-919.

4. Churg, A., Warnock, M.L. \& Bensch, K.G. Malignant mesothelioma arising after direct application of asbestos and fiberglass to the pericardium. Am Rev Resp Dis 1978, 118: 419-424.

5. Kahn, E.I., Rohl, A., Barrett, E.W. \& Suzuki, Y. Primary pericardial mesothelioma following exposure to asbestos. Env Res 1980, 23: 270-281.

6. Ordóñez, N.G. The immunohistochemical diagnosis of mesothelioma. Am J Surg Path 1989, 13: 276-291. chemistry, A. Walters for photographic help, D. Anglin for typing the manuscript, and the staff of the Medical Library, St Mary's Hospital Medical School.

7. Shin, M.S., Ho, K.-J. \& Liu, L.B. Pericardial mesothelioma masquerading as rheumatic heart disease. Arch Intern Med 1977, 137: 257.

8. Lund, O., Hansen, O.K., Ardest, S. \& Baandrup, U. Primary malignant pericardial mesothelioma mimicking left atrial myxoma. Scand J Thor Cardiovasc Surg 1987, 21: 273-275.

9. Llewellyn, M.J., Atkinson, M.W. \& Fabri, B. Pericardial constriction caused by primary mesothelioma. Br Heart $J$ 1987, 57: 54-57.

10. Bertrand, E. Cardiovascular disease. In: Manson-Bahr, P.E.C. \& Bell, D.R. (eds) Manson's Tropical Diseases, 19th ed. Baillière Tindall, London, 1987, p. 1026.

11. Narayanan, P.S., Chandrasekar, S. \& Madhavan, M. Intrathoracic mesotheliomas associated with tuberculosis. Indian J Med Sci 1972, 26: 432-436. 